\title{
MONODROMIES OF ALGEBRAIC CONNECTIONS ON THE TRIVIAL BUNDLE
}

\author{
BYUNGHEUP JUN
}

\begin{abstract}
In this note, we study monodromies of algebraic connections on the trivial vector bundle. We prove that on a smooth complex affine curve, any monodromy arises as the underlying local system of an algebraic connection on the trivial bundle. We give a generalization of this for rank one monodromies in higher dimension.
\end{abstract}

\section{Monodromies des connexions algébriques sur le fibré triv- ial \\ RÉSUMÉ. Dans cette note, nous étudions la monodromie de connexions algébriques sur le fibré vectoriel trivial. Nous prouvons que sur une courbe affine, lisse, complexe, toute monodromie est sous-jacente à une connexion algébrique sur le fibré trivial. Nous donnons une généralisation de ceci aux monodromies de rang un en dimension supérieure.}

\section{VERSION FRANÇAISE ABRÉGÉE}

Soit $U$ une variété affine lisse sur $\mathbb{C}$. Deligne a montré dans [3] qu'il y a une correspondence biunivoque entre les sytèmes locaux sur $U$ et les fibrés vectoriels algébriques à connexion plate, régulière singulière à l'infini. Si on supprime la condition de régularité à l'infini, alors il y a beaucoup de connexions plates algébriques à monodromie fixée. Dans 四, il prouve que si $\operatorname{dim} U=1$, toute monodromie de rang 1 est sous-jacente à une connexion algébrique sur le fibré trivial. Dès lors, les singularités à l'infini peuvent être irrégulières.

Théorème 1 (Deligne [⿶). Sur une courbe affine lisse complexe $U$, tout caractère $\rho$ du groupe fondamental $\pi_{1}\left(U_{\mathrm{an}}\right)$ est sous-jacent à une connexion algébrique sur le fibré trivial.

Idée de la preuve.- On identifie $H_{d R}^{1}(U)=H^{1}\left(U_{\text {an }}, \mathbb{C}\right)([5])$, ce qui associe à la classe de de Rham de $\omega$ dans $\Gamma\left(U, \Omega_{U}^{1}\right)$ son exponentielle $\exp [\omega]$ dans $H^{1}\left(U_{\text {an }}, \mathbb{C}^{*}\right)$, qui correspond à la monodromie sous-jacente à la connexion $(\mathcal{O}, d-\omega)$. L'annulation de $H^{2}\left(U_{\text {an }}, \mathbb{Z}\right)$ permet de conclure.

c.q.f.d.

Nous donnons une version légr̀ement différente du théorème 1 dans le corollaire suivant.

Corollaire 2. Soit $U$ une variété affine lisse complexe. Les conditions suivantes sont équivalentes.

1) $H^{2}\left(U_{\mathrm{an}}, \mathbb{Z}\right)$ n'a pas de torsion.

2) Tout caractère $\rho$ du groupe fondamental $\pi_{1}\left(U_{\text {an }}\right)$ est sous-jacent à une connexion algébrique sur le fibré trivial.

Date: 25. June, 2000. 
3) Soit $\mathcal{L}$ un fibré algébrique de rang 1 avec classe de Chern triviale dans $H_{d R}^{2}(U)$. Alors tout caractère $\rho$ du groupe fondamental $\pi_{1}\left(U_{\text {an }}\right)$ est sous-jacent à une connexion algébrique sur $\mathcal{L}$.

Dans le théorème suivant, nous donnons un exemple d'application.

Théorème 3. Soit $X$ une variété projective lisse complexe et soit $D$ un diviseur ample supportant le groupe de Néron-Severi $N S(X)$ de X. Alors tout caractère $\rho$ du groupe fondamental $\pi_{1}\left(U_{\mathrm{an}}\right)$ est sous-jacent à une connexion algébrique sur le fibré trivial.

Nous renvoyons au diagramme (5) de Remark 1 dans $\S 2$ pour une visualisation des résultats précédents.

Soit $U$ de nouveau une courbe lisse affine complexe. Nous généralisons le théorème 1 aux représentations de rang supérieur. Nous partons de la décomposition d'une représentation $\rho$ à valeurs dans $\mathrm{GL}(r)$ comme produit tensoriel $\chi \otimes \phi$, où $\chi$ est un caractère et $\phi$ est une représentation à valeurs dans $S L(r)$.

Nous considérons la suite exacte

$$
0 \rightarrow \mu_{r} \rightarrow \mathbb{G}_{m} \times \mathrm{SL}(r) \stackrel{\pi}{\rightarrow} \mathrm{GL}(r) \rightarrow 0,
$$

où $\mu_{r}$ est le groupe des racines $r$-ièmes de l' unité, et où la première application est définie par $\zeta \mapsto(\zeta,(1 / \zeta) I)$ et la seconde est simplement la multiplication d'une matrice par un scalaire.

Alors on a la suite exacte longue de cohomologie sur $U_{\text {an }}$ (d'ensembles pointés)

$$
\cdots \rightarrow H^{1}\left(U_{\mathrm{an}}, \mathbb{G}_{m} \times \mathrm{SL}(r)\right) \stackrel{\pi_{*}}{\rightarrow} H^{1}\left(U_{\mathrm{an}}, \mathrm{GL}(r)\right) \stackrel{\delta}{\rightarrow} H^{2}\left(U_{\mathrm{an}}, \mu_{r}\right) \rightarrow \cdots
$$

Le groupe $H^{2}\left(U_{\mathrm{an}}, \mu_{r}\right)$ est nul car on est en dimension 1. Donc $\pi_{*}$ est surjective. Par conséquent, il suffit de prouver le théorème pour des représentations à déterminant trivial.

Proposition 4. Toute représentation $\rho: \pi_{1}\left(U_{\text {an }}\right) \rightarrow S L(r, \mathbb{C})$ du groupe fondamental est sous-jacente à une connexion algébrique sur le fibré trivial.

Idée de la preuve.- Soit $\rho: \pi_{1}\left(U_{\text {an }}\right) \rightarrow S L(r, \mathbb{C})$ et soit $(E, \nabla)$ l'unique fibré à connexion régulière singulière à l'infini. Sur une courbe affine $U$, un fibré algébrique $E$ a une décomposition $\mathcal{O}^{r-1} \oplus \mathcal{L}$ (cf. [1], [2]). Le déterminant $\operatorname{det}(E)=\mathcal{L}$ a la connexion déterminant, qui est aussi régulière singulière à l' infini. Comme $\operatorname{det} \rho=1$, on a $(\operatorname{det}(E), \operatorname{det}(\nabla))=\left(\mathcal{O}_{U}, d\right)$, donc en particulier $\operatorname{det}(E)=\mathcal{L}=\mathcal{O}$. c.q.f.d.

Finalement, nous obtenons

Théorème 5. Soit $U$ une courbe affine lisse complexe. Alors, toute représentation $\rho: \pi_{1}\left(U_{\text {an }}\right) \rightarrow G L(r, \mathbb{C})$ du groupe fondamental est sous-jacente à une connexion algébrique sur le fibré trivial.

\section{INTRODUCTION}

On a nonsingular projective algebraic variety $X$ over $\mathbb{C}$, using the existence theorem of Cauchy-Kovalevski and Serre's GAGA( $[\sqrt{6}])$, we have the Riemann-Hilbert correspondence: there exists a unique algebraic vector bundle with a connection for a given representation of the fundamental group $\pi_{1}(X)$ and vice versa. 
On a noncomplete variety, the uniqueness no longer holds: there exist several vector bundles with connection for a monodromy. In [3], Deligne showed the existence and uniqueness with regularity condition at ininity.

Instead of regularity, in this article, we think about a restriction of the underlying vector bundle of the connection. In [4], Deligne showed one can realize any monodromy representation of the fundamental group of an affine curve $U$ as the underlying monodromy of a connection $\nabla=d-\omega$ on the trivial bundle of rank 1 on $U\left(\right.$ ie. $\left.\mathcal{O}_{U}\right)$.

The main theorem(Theorem 5) generalizes Deligne's result(Theorem 1): On an affine curve, to any monodromy representation of the fundamental group, there is a connection on trivial bundle with the prescribed underlying monodromy.

The aim of this note is to introduce Deligne's result on rank 1 case in and to generalize it in two directions: one is when $U$ is of higher dimension and the other is the similar to the higher rank representation of the fundamental group of the affine curve.

Acknowledgements. Theorem 1, on which this note relies, is essentially due to Pierre Deligne.(顿). I thank to Hélène Esnault for all the fruitful discussions on of my work and for sharing her time and ideas.

\section{RANK 1 REPRESENTATIONS}

Theorem 1 (Deligne [4]). On a smooth affine curve $U$, to given rank 1 monodromy representation $\rho$ of $\pi_{1}\left(U_{\text {an }}\right)$, there exists an algebraic connection $\nabla$ on $\mathcal{O}$ with the underlying monodromy $\rho$.

Proof. From the exponential sequence, we have the following exact sequence of singular cohomology groups.

$$
H^{1}\left(U_{\mathrm{an}}, \mathbb{C}\right) \stackrel{\exp }{\longrightarrow} H^{1}\left(U_{\mathrm{an}}, \mathbb{C}^{*}\right) \longrightarrow H^{2}\left(U_{\mathrm{an}}, \mathbb{Z}\right) \longrightarrow H^{1}\left(U_{\mathrm{an}}, \mathbb{C}\right),
$$

where $U_{\text {an }}$ denotes $U$ with the strong topology. We know $H^{1}\left(U_{\text {an }}, \mathbb{C}^{*}\right)$ is the group of isomorphism classes of rank 1 local systems on $U_{\text {an }}$ and $H^{2}\left(U_{\text {an }}, \mathbb{Z}\right)$ vanishes by dimension reason.

Via the identification(cf. [5])

$$
H_{d R}^{*}(U):=\mathbb{H}^{*}\left(U, \Omega^{\bullet}\right)=H^{1}\left(U_{\text {an }}, \mathbb{C}\right),
$$

the de Rham class of $\omega$ in $H^{0}\left(U, \Omega^{1}\right)$ yields a class $[\omega]$ in $H^{1}\left(U_{\text {an }}, \mathbb{C}\right)$, such that

$$
\exp [\omega] \in H^{1}\left(U_{\text {an }}, \mathbb{C}^{*}\right)=\operatorname{Hom}\left(\pi_{1}\left(U_{\text {an }}\right), \mathbb{C}^{*}\right)
$$

is the underlying monodromy of $(\mathcal{O}, d-\omega)$.

On an affine variety, the higher coherent sheaf cohomology vanishes. Thus $H^{0}\left(U, \Omega^{1}\right)$ generates $H_{d R}^{1}(U)$. Therefore $H^{0}\left(U, \Omega^{1}\right)$ surjects onto $\operatorname{Hom}\left(\pi_{1}\left(U_{\text {an }}\right), \mathbb{C}^{*}\right)$. This finishes the proof.

q.e.d.

Without much difficulty, we generalize the theorem when $\operatorname{dim} U>1$.

Corollary 2. Let $U$ be an affine variety over $\mathbb{C}$. Then the followings are equivalent:

1) $H^{2}\left(U_{\mathrm{an}}, \mathbb{Z}\right)$ is torsion-free.

2) To any rank 1 representation $\rho$ of $\pi_{1}\left(U_{\mathrm{an}}\right)$, there exists an algebraic connection $\nabla$ on $\mathcal{O}$ with underlying monodromy $\rho$. 
3) If a line bundle $\mathcal{L}$ has vanishing Chern class in $H_{d R}^{2}(U)$. To any rank 1 representation $\rho$ of $\pi_{1}\left(U_{\mathrm{an}}\right)$ and, there exists an algebraic connection $\nabla$ on $\mathcal{L}$ with underlying monodromy $\rho$.

Proof. 1) $\Leftrightarrow 2$ ) As in the proof of the theorem 1, one has the exact sequence arising from the exponential sequence $(3)$. On an affine variety $U, \Gamma\left(U, \Omega_{\text {closed }}^{1}\right)$ generates $H_{d R}^{1}(U)=H^{1}\left(U_{\text {an }}, \mathbb{C}\right)$. Then exp in the exact sequence is surjective if and only if $\Gamma\left(U, \Omega_{\text {closed }}^{1}\right) \rightarrow H^{1}\left(U_{\text {an }}, \mathbb{C}^{*}\right)$ is surjective or equivalently, $H^{2}\left(U_{\text {an }}, \mathbb{Z}\right)$ is torsion-free.

$2) \Rightarrow 3$ ) Consider the complex of sheaves on the Zariski topology of $U$ :

$$
\mathcal{C}^{\bullet}: \mathcal{O}^{*} \stackrel{d \log }{\longrightarrow} \Omega^{1} \rightarrow \Omega^{2} \rightarrow \cdots
$$

The first hypercohomology of $\mathcal{C}^{\bullet}$ is the group of isomorphism classes of line bundles with integrable connection, which will be denoted by $\operatorname{Pic}^{\nabla}(U)$. The filtration of $\mathcal{C}$ by its degree gives

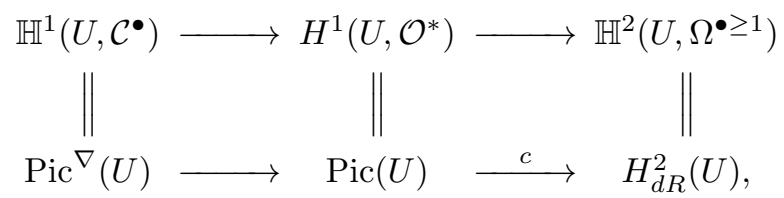

where $c$ denotes the first Chern class map and the last vertical map is an isomorphism since the kernel and the cokernel are $H^{1}(U, \mathcal{O})=H^{2}(U, \mathcal{O})=0$.

Since $c(\mathcal{L})=0$, from the exact sequence, there exists an integrable connection $\nabla_{0}$ on $\mathcal{L}$. Let $\rho_{0}$ be the monodromy of $\left(\mathcal{L}, \nabla_{0}\right)$. As one can realize any monodromy on $\mathcal{O}$, one has an algebraic connection $\nabla_{1}$ with the monodromy $\rho_{0}^{-1} \cdot \rho$ on $\mathcal{O}$. Twisting $\left(\mathcal{L}, \nabla_{0}\right)$ with $\left(\mathcal{O}, \nabla_{1}\right)$, one has the connection $\nabla=\nabla_{0} \otimes \nabla_{1}$ on $\mathcal{L}$ with the desired monodromy $\rho$.

3) $\Rightarrow$ 2) Clear.

q.e.d.

As a special case of the corollary, we have the following:

Theorem 3. Let $X$ be a smooth projective variety over $\mathbb{C}$ and $D$ be an ample divisor supporting the Néron-Severi group $N S(X)$ of $X$. Then to any monodromy $\rho$ in $\operatorname{Hom}\left(\pi\left(U_{\mathrm{an}}\right), \mathbb{C}^{*}\right)$, there exists an integrable algebraic connection $\nabla=d-\omega$ on $\mathcal{O}_{U}$ with underlying monodromy $\rho$.

Proof. By the equivalence of the corollary 2, we have to show that $H^{2}\left(U_{\text {an }}, \mathbb{Z}\right)$ is torsion-free. But, in the exact sequence (3), torsion comes from $H^{1}\left(U_{\text {an }}, \mathbb{C}^{*}\right)$. Using the existence theorem in [3], one has a line bundle on $X$ with connection $(\mathcal{L}, \nabla)$ which is regular singular at $D$ with the underlying monodromy $\rho$. The image of $\mathcal{L}$ maps into $N S(X)$, and thus a torsion element in $H^{2}\left(U_{\text {an }}, \mathbb{Z}\right)$ vanishes. Therefore $H^{2}\left(U_{\text {an }}, \mathbb{Z}\right)$ is torsion-free.

q.e.d.

Remark 1. Using a commutative diagram, one can summarize the result in a simple form where all rows and columns are exact: 
$(5)$

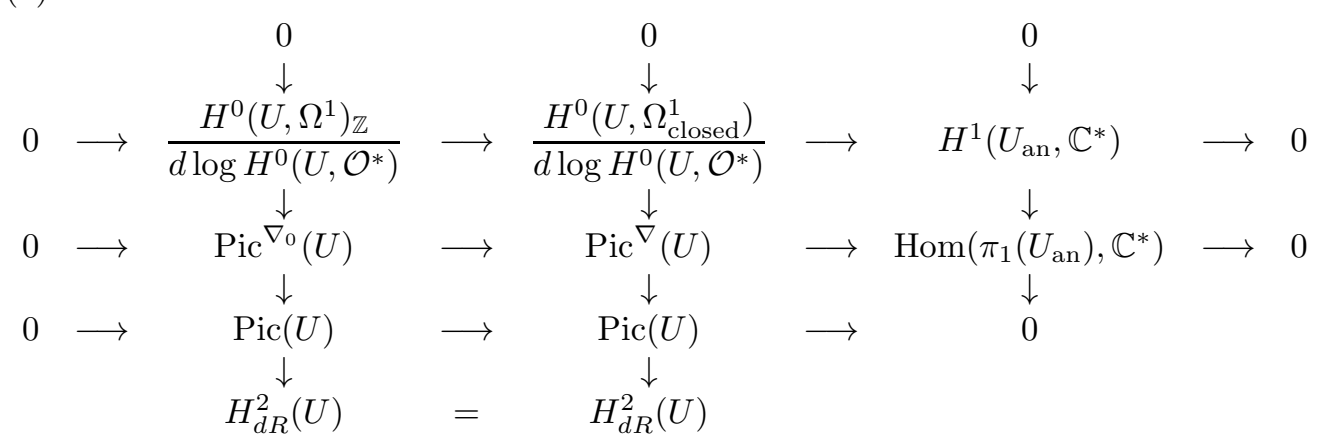

In the diagram, $\operatorname{Pic}^{\nabla_{0}}(U)$ is the subgroup of $\operatorname{Pic}^{\nabla}(U)$ with respect to the trivial monodromy and $H^{0}\left(U, \Omega^{1}\right)_{\mathbb{Z}}$ is the global 1-forms with integral periods. When $\operatorname{dim} U=1$, one has $H_{d R}^{2}(U)=0$. Thus, we have $\operatorname{Pic}^{\nabla}(U)$ and $\operatorname{Pic}^{\nabla_{0}}(U)$ as extensions of the $\operatorname{Pic}(U)$.

\section{Higher RANK CASE ON CURVES}

In this section, $U$ is again a smooth affine curve over $\mathbb{C}$.

One has the short exact sequence:

$$
\begin{gathered}
0 \rightarrow \mu_{r} \rightarrow \mathbb{G}_{m} \times \mathrm{SL}(r) \stackrel{\pi}{\rightarrow} \mathrm{GL}(r) \rightarrow 0, \\
\zeta \mapsto(\zeta,(1 / \zeta) I) \\
(\ell, M) \mapsto \ell M
\end{gathered}
$$

where $\mu_{r}$ denotes the multiplicative group of $r$-th roots of unity. Because $\mu_{r}$ maps into the center of $\mathbb{G}_{m} \times \mathrm{SL}(r)$, we have long exact sequence of cohomologies on $U_{\text {an }}$ (of pointed sets).

$$
\cdots \rightarrow H^{1}\left(U_{\mathrm{an}}, \mathbb{G}_{m} \times \mathrm{SL}(r)\right) \stackrel{\pi_{*}}{\rightarrow} H^{1}\left(U_{\mathrm{an}}, \mathrm{GL}(r)\right) \stackrel{\delta}{\rightarrow} H^{2}\left(U_{\mathrm{an}}, \mu_{r}\right) \rightarrow \cdots
$$

By dimension reason, $H^{2}\left(U_{\mathrm{an}}, \mu_{r}\right)$ vanishes and one sees $\pi_{*}$ surjective. Thus it suffices to solve the problem for $S L$-representations.

If $A$ is a Dedekind domain and $M$ is a projective $A$-module of finite rank $r$, one has $M \cong A^{r-1} \oplus L$ where $r=\operatorname{rank}(M)$ and $L \in \operatorname{Pic}(A)$. As a special case, a vector bundle $E$ of $\operatorname{rank}(E)=r$ on an affine curve $U$ is isomorphic to $\mathcal{O}^{r-1} \oplus \mathcal{L}$, where $\mathcal{L}=\operatorname{det} E$ is a line bundle on $U$ (cf. [2], [1])

Proposition 4. Let $U$ be a smooth affine curve. To any $\mathrm{SL}(r)$-representation $\rho$ of $\pi_{1}\left(U_{\mathrm{an}}\right)$, there exists an algebraic connection $\nabla$ on the trivial bundle of rank $r$ with underlying monodromy $\rho$.

Proof. Let $\rho$ be a SL $(r)$-representation and $X=U \cup D$ be the smooth completion of $U$. Then there exists a vector bundle $E$ on $X=U \cup D$ the smooth completion of $U$, with a connection $\nabla$ which is regular singular,

$$
\nabla: E \rightarrow E \otimes \Omega^{1}(\log D)
$$

such that the restriction on $U$ gives the monodromy $\rho$ (cf. [3]).

By the decomposition theorem, one has $\left.E\right|_{U}=\mathcal{O}_{U}^{r-1} \oplus \mathcal{L}$, where $\mathcal{L}=\operatorname{det}\left(\left.E\right|_{U}\right)$. On $X$,

$$
\operatorname{det} \nabla: \operatorname{det}(E) \rightarrow \operatorname{det}(E) \otimes \Omega^{1}(\log D)
$$


is regular at $D$ and has trivial monodromy since the representation is $S L$-valued. Using the uniqueness theorem in [3], we have

$$
\left(\left.\operatorname{det}(E)\right|_{U}, \operatorname{det} \nabla\right)=\left(\mathcal{O}_{U}, d\right) .
$$

In particular $\mathcal{L}=\mathcal{O}_{U}$ and $E_{U}=\mathcal{O}_{U}^{r}$.

q.e.d.

After the proposition, we obtain the main theorem.

Theorem 5. Let $U$ be a smooth affine curve over $\mathbb{C}$. Then to any rank $r$ representation of the fundamental group $\rho \in \operatorname{Hom}\left(\pi_{1}\left(U_{\mathrm{an}}\right), \mathrm{GL}(r)\right)$, there exists a connection on the trivial bundle of rank $r$ on an affine open set $U$ with underlying monodromy $\rho$.

Proof. Clear.

q.e.d.

\section{REFERENCES}

[1] Atiyah, M. F.: Vector bundles over an elliptic curve, Proc. London Math. Soc. (3) 7 (1957) 414-452.

[2] Bourbaki, N.: Éléments de mathématique, Algèbre commutative, Masson, Paris (1983).

[3] Deligne, P.: Équations différentielles à points singuliers réguliers, Springer LN 163 (1970).

[4] Deligne, P.: Letter to H. Esnault, Apr. 22. (1999).

[5] Grothendieck, A.: On the de Rham cohomology of algebraic varieties, Publ. Math. IHES 29 (1966), 95-103.

[6] Serre, J.-P.: Géométrie algébrique et géométrie analytique, Ann. Inst. Fourier. 6 (1955-1956), $1-42$.

E-mail address: byungheup.jun@uni-essen.de

FB 6, Mathematik, Universität Essen, 45117 Essen, Germany 